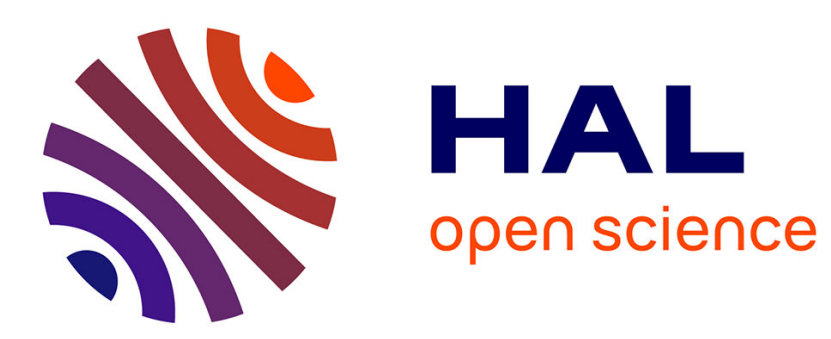

\title{
Extension of Popov Absolute Stability Criterion to Nonautonomous Systems with Delays
}

\author{
Pierre-Alexandre Bliman
}

\section{To cite this version:}

Pierre-Alexandre Bliman. Extension of Popov Absolute Stability Criterion to Nonautonomous Systems with Delays. [Research Report] RR-3625, INRIA. 1999. inria-00073052

\section{HAL Id: inria-00073052 \\ https://hal.inria.fr/inria-00073052}

Submitted on 24 May 2006

HAL is a multi-disciplinary open access archive for the deposit and dissemination of scientific research documents, whether they are published or not. The documents may come from teaching and research institutions in France or abroad, or from public or private research centers.
L'archive ouverte pluridisciplinaire HAL, est destinée au dépôt et à la diffusion de documents scientifiques de niveau recherche, publiés ou non, émanant des établissements d'enseignement et de recherche français ou étrangers, des laboratoires publics ou privés. 


\section{Extension of Popov absolute stability criterion to nonautonomous systems with delays.}

Pierre-Alexandre Bliman

$\mathbf{N}^{\circ} \mathbf{3 6 2 5}$

Février 1999

THÈME 4 



\title{
Extension of Popov absolute stability criterion to nonautonomous systems with delays.
}

\author{
Pierre-Alexandre Bliman* \\ Thème 4 - Simulation et optimisation \\ de systèmes complexes \\ Projet Sosso
}

Rapport de recherche $\mathrm{n}^{\circ} 3625$ - Février 1999 - 20 pages

\begin{abstract}
This paper extends in a simple way the classical absolute stability Popov criterion to multivariable systems with delays and with time-varying memoryless nonlinearities subject to sector conditions. The proposed sufficient conditions are expressed in the frequency domain, a form well-suited for robustness issues, and lead to simple graphical interpretations for scalar systems. Apart from the usual conditions, the results assume basically a generalized sector condition on the derivative of the nonlinearities with respect to time. Results for local and global stability are given, the latter concerning in particular the linear time-varying ones. For rational transfers, the frequency conditions are equivalent to some easy-to-check Linear Matrix Inequalities: this leads to a tractable method of numerical resolution by approximation. As an illustration, a numerical example is provided.
\end{abstract}

Key-words: Delay systems, absolute stability, Popov criterion, time-varying nonlinearities, frequency domain, nonlinear systems, multivariable systems, robustness margin.

(Résumé : tsvp)

* email: pierre-alexandre.bliman@inria.fr

Unité de recherche INRIA Rocquencourt

Domaine de Voluceau, Rocquencourt, BP 105, 78153 LE CHESNAY Cedex (France)

Téléphone : 0139635511 - International : +33139635511

Télécopie : (33) 0139635330 - International : +331396353 30 


\section{Extension du critère de Popov de stabilité absolue aux systèmes non-autonomes à retards.}

Résumé : Ce rapport étend de manière simple le classique critère de stabilité absolue de Popov aux systèmes multivariables comportant des retards et des non-linéarités sans mémoire et dépendant du temps, soumises à des conditions de secteur. Les conditions suffisantes que nous proposons sont exprimées dans le domaine fréquentiel, une forme adaptée à l'étude de la robustesse, et conduisent à des interprétations graphiques simples pour les systèmes scalaires. En plus d'hypothèses habituelles, les résultats supposent essentiellement la vérification d'une condition de secteur généralisée, portant sur la dérivée par rapport au temps de la non-linéarité. Des résultats de stabilité locale et globale sont donnés, ces derniers concernant en particulier les systèmes linéaires instationnaires. Les conditions fréquentielles à vérifier sont équivalentes, pour des transferts rationnels, à des systèmes d'inégalités linéaires matricielles ( $L M I$ en anglais) faciles à vérifier: ceci conduit à une méthode implémentable de résolution numérique par approximation. Un exemple numérique illustre les résultats.

Mots-clé : Systèmes à retards, stabilité absolue, critère de Popov, non-linéarités dépendant du temps, domaine fréquentiel, systèmes non linéaires, systèmes multivariables, marge de robustesse. 


\section{Introduction}

This paper deals with an extension of Popov absolute stability criterion to nonstationary delay systems. We consider the multivariable control system given in Figure 1, where $H$ is a strictly proper transfer function matrix of size $p \times p, p \in \mathbb{N} \backslash\{0\}$, and $\psi: \mathbb{R} \times \mathbb{R}^{p} \rightarrow \mathbb{R}^{p}$ a time-dependent nonlinearity.

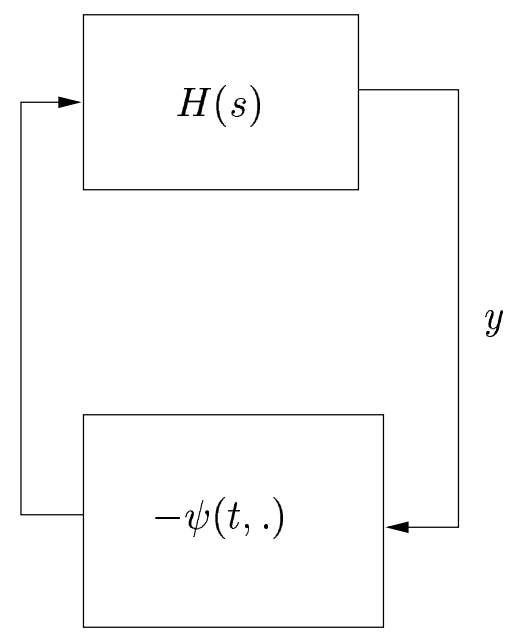

Figure 1: The system under study

Here, the transfer function matrix $H$ is supposed to be represented by the following delay differential system

$$
\dot{x}=\sum_{l=0}^{L} A_{l} x\left(t-h_{l}\right)+B u, \quad u=-\psi(t, y), \quad y=\sum_{l=0}^{L} C_{l} x\left(t-h_{l}\right),\left.\quad x\right|_{[-h, 0]}=\phi
$$

where

$$
n \in \mathbb{N} \backslash\{0\}, L \in \mathbb{N}, x \in \mathbb{R}^{n}, y \in \mathbb{R}^{p}, A_{l} \in \mathbb{R}^{n \times n}, B \in \mathbb{R}^{n \times p}, C_{l} \in \mathbb{R}^{p \times n}, 0 \leq h_{l} \leq h,
$$

in such a way that

$$
H(s)=\left(\sum_{l=0}^{L} C_{l} e^{-h_{l} s}\right)\left(s I-\sum_{l=0}^{L} A_{l} e^{-h_{l} s}\right)^{-1} B .
$$

Assume that $\psi$ is not perfectly known, but rather that it belongs to a certain class of nonlinearities, defined by the following properties: $\psi$ is decentralized [15] (that is: $\forall i \in\{1, \ldots p\}, \psi_{i}(t, y)=\psi_{i}\left(t, y_{i}\right)$ ), time-invariant, and verifies moreover the following sector condition

$$
\forall y \in \mathbb{R}^{p}, \quad \psi(y)^{T}(\psi(y)-K y) \leq 0,
$$

for a certain nonnegative diagonal matrix $K$. The asymptotic stability of all the systems obtained by coupling (1) with a nonlinearity fulfilling (2) is called absolute stability of the class of systems (1).

In order for the class of systems (1) to be absolutely stable, asymptotic stability should hold for all linear time-invariant choice of $\psi$ compatible with (2), that is for the maps:

$$
\psi(t, y)=\operatorname{diag}\left\{k_{i}\right\} y, \quad 0 \leq k_{i} \leq K_{i} .
$$

In 1947, Aizerman [1] was the first to formulate (for finite sectors and rational systems) the question of the sufficiency of this condition. As is well-known, the answer is negative, even if one restricts oneself to 
time-invariant nonlinearities, as proved in 1958 by Pliss [24] (see also a counterexample in [2, p. 86-88]). Popov gave in 1959 an elegant sufficient condition for absolute stability of the class of systems (1) with time-invariant nonlinearities [25], known as Popov criterion (see e.g. [37, 15, 17]): absolute stability holds, provided that the poles of $H$ have negative real part and that there exists a diagonal matrix $\eta$ such that

$$
I+(I+\eta s) K H(s) \quad \text { is strictly positive real (SPR) . }
$$

The latter result was initially given for rational systems, and it was extended shortly after by Popov et al. [26] to delay systems (and seemingly independently by $\mathrm{Li}[18]$ ). For rational systems, the result is proved using a Lyapunov function with a Lur'e term, of the form

$$
V(t, x) \stackrel{\text { def }}{=} x^{T} P x+2 \sum_{i=1}^{p} \eta_{i} K_{i} \int_{0}^{y_{i}} \psi_{i}\left(t, z_{i}\right) d z_{i} .
$$

Since the late fifties, a lot of contributions have been published, giving generalizations of Popov criterion to various classes of time-invariant nonlinearities (see [17, 4] for an overview and references, [19] for chinese contributions). As an example, the result has been extended by Yakubovich to some hysteresis nonlinearities [38]. Results have been obtained with stronger conditions on the nonlinearity, especially incremental sector conditions, see references in $[22,17]$.

Also, attempts have been made to adapt Popov criterion to time-varying rational systems (see [20] for a review of the period 1968-1977, surveying an important number of contributions, especially from Eastern Europe). Pyatnitskii has shown [27] that absolute stability for a class of time-varying nonlinearities fulfilling the sector condition

$$
\forall(t, y) \in \mathbb{R}^{+} \times \mathbb{R}^{p}, \quad \psi(t, y)^{T}(\psi(t, y)-K y) \leq 0,
$$

is equivalent to the asymptotic stability of all the time-varying linear systems of this class, that is for the maps

$$
\psi(t, y)=\operatorname{diag}\left\{k_{i}(t)\right\} y, \quad 0 \leq k_{i}(t) \leq K_{i} .
$$

This result gives rise to a class of sufficient conditions of absolute stability, see e.g. [21]. See also [16, 3] for some frequential conditions of absolute stability without restrictions on the rate of variation of the nonlinearity.

On the other hand, it is possible to obtain conditions of absolute stability for smaller classes of timevarying nonlinearities, especially by making restrictions on $\frac{\partial \psi}{\partial t}$. This limitation may be acceptable e.g. when studying the stability of limit cycles. Narendra et al. devoted Chapter VI of their monograph [22] to this question. They obtained conditions for global stability involving two parts: the Popov condition plus a differential (in the case of a so-called separate nonlinearity $\psi(t, y)=k(t) f(y)$ ) or integrodifferential inequality, linking $\frac{\partial \psi}{\partial t}$ and $\psi$. These conditions may be not so easy to handle, see examples in [22, Chapter VIII]. In [36], Walker provided conditions for global stability. The assumptions given therein imply that (3) holds and, e.g. when $\eta \geq 0$,

$$
\forall(t, y) \in \mathbb{R}^{+} \times \mathbb{R}^{p}, \quad \psi(t, y)^{T}(\psi(t, y)-K y) \leq-\eta K \frac{d}{d t}\left[\int_{0}^{y} \psi(t, z) d z\right],
$$

instead of (2). As the a priori knowledge on the right-hand side does not usually permit to consider it as nonnegative, this expresses a restriction of the rate of variation of the nonlinearity, but $K$ does not define anymore the width of the sector, and it is not clear how to check systematically the conditions, especially for multivariable systems. Rekasius et al. [32], Hul'chuk et al. [14] and Bertoni et al. [5] published contributions providing frequency criteria for absolute stability of nonstationary systems. In [32, 14], the authors request for the terms of the form $\int_{0}^{y_{i}} \frac{\partial \psi_{i}}{\partial t}(t, z) d z$ appearing in the derivative of the Lyapunov function (4) to be bounded by a quadratic form in $y_{i}$ and $\psi_{i}\left(t, y_{i}\right)$. This condition, see (6) below, may be interpreted as a generalized sector condition; it is fulfilled e.g. when a sector condition on $\frac{\partial \psi}{\partial t}(t, y)$ holds. In [5], only this simpler condition is used, and graphical interpretation is given. See also [11] for related approach. 
To the best of our knowledge, very few papers have been published on the topic of absolute stability of time-varying delay systems. Some non-frequential criteria are cited in [19, Paragraphs 6.1 and 6.5]. A paper by Răsvan [30] provides an extension of Popov criterion to systems with separate nonlinearities. Achieving an analysis close to the one presented here, it has to assume the monotonicity (wrt time) of the time varying gains, a quite limiting assumption. Also, Walker [35] provides results generalizing the approach of [36] to delay systems.

In the present paper, one proposes an extension of Popov criterion to nonautonomous systems with delays, generalizing the work done in [6] for rational systems, in the spirit of $[5,14,32]$. More precisely, one provides simple conditions ensuring uniform asymptotic stability of the origin. These conditions are expressed in terms of a frequency condition in Theorems 1 and 3. The results provide uniform local asymptotic stability, a generalization of the property of absolute stability with finite domain [15], or global stability. The latter results may be applied in particular to linear time-varying operators $\psi$. The proposed criteria just add some supplementary terms to Popov criterion, depending on $\frac{\partial \psi}{\partial t}(t, y)$ as in $[32,14]$. The results, being expressed in the frequency domain, are well fitted to robustness issues, especially in presence of unstructured perturbations. Some graphical interpretations are provided for scalar systems, partly as in [5]. Concerning checkability of the conditions, an attracting feature is the possibility to approximate the transfer function matrix $H$ by rational transfers: it is shown in [6] that for these systems, the proposed frequency conditions are equivalent to some Linear Matrix Inequalities, a now standard class of problems for which sound numerical methods have been developed [7].

An example of application of the results given here comes from the control of chaos [10]: in order to stabilize an unstable periodic orbit of a strange attractor, Pyragas [28, 29] proposed to use a feedback control built on the difference between the actual value and the delayed value of the output, with delay equal to the period of the cycle.

Finally, we want to emphasize the fact that the results could be applied to more general systems (e.g. systems with distributed delays, integral systems), as it is indeed the case for Popov criterion, see [12, §4.6.] and $[8]$.

The paper is organized as follows. The criteria are stated in Section 2. Computation issues are studied in Section 3. An example is presented in Section 4. Finally, proofs of the results are exposed in Section 5.

Notations In all the paper, $\|\cdot\|$ denotes the euclidian norm or the induced matrix norm, $I_{r}$ denotes the $r \times r$ identity matrix (simply $I$ when the context is clear), the asterisk * denotes complex conjugation. For any real diagonal matrix $\eta=\operatorname{diag}\left\{\eta_{i}\right\}$, one denotes

$$
|\eta| \stackrel{\text { def }}{=} \operatorname{diag}\left\{\left|\eta_{i}\right|\right\}, \quad \operatorname{sgn} \eta \stackrel{\text { def }}{=} \operatorname{diag}\left\{\operatorname{sgn} \eta_{i}\right\},
$$

where one may take indifferently $\operatorname{sgn} 0=-1$ or +1 . When $\eta$ is a function of $t \in \mathbb{R}^{+}$, by definition:

$$
\sup _{t \geq 0} \eta(t) \stackrel{\text { def }}{=} \operatorname{diag}\left\{\sup _{t \geq 0} \eta_{i}(t)\right\}
$$

and similarly for the infimum, the essential supremum, ... and so on. For $z \in \mathbb{R}$, one denotes

$$
|z|_{+} \stackrel{\text { def }}{=} \sup \{z, 0\}, \quad|z|_{-} \stackrel{\text { def }}{=} \sup \{-z, 0\} .
$$

The same notation is used for diagonal matrices:

$$
|\eta|_{ \pm}=\sup \{ \pm \eta, 0\}=\operatorname{diag}\left\{\sup \left\{ \pm \eta_{i}, 0\right\}\right\}=\operatorname{diag}\left\{\left|\eta_{i}\right|_{ \pm}\right\}
$$

\section{Main results}

In all the sequel, we assume that there exist global solutions of (1), that is, by definition: for all $\phi \in$ $\mathcal{C}\left([-h, 0] ; \mathbb{R}^{n}\right)$, there exists a continuous function $x$ defined on $[-h,+\infty)$, absolutely continuous [34] on $[0,+\infty)$, such that $\left.x\right|_{[-h, 0]}=\phi$ and (1) is fulfilled almost everywhere on $[0,+\infty)$. The stability results given below concern the asymptotic behavior of these global solutions. 
Theorem 1 (A frequency criterion). Assume that there exists a convex open neighborhood $\mathcal{O}$ of 0 in $\mathbb{R}^{p}$ for which the following assumptions hold.

(H0) The function $\psi$ is measurable and, for any $y \in \mathcal{O}, t \mapsto \psi(t, y)$ is locally Lipschitz (and hence t-a.e. differentiable), with a Lipschitz constant locally integrable wrt $y \in \mathcal{O}$.

(H1) The nonlinearity $\psi$ is decentralized and there exists a diagonal matrix $K=\operatorname{diag}\left\{K_{i}\right\} \geq 0$ such that,

$$
\forall(t, y) \in \mathbb{R}^{+} \times \mathcal{O}, \quad \psi(t, y)^{T}(\psi(t, y)-K y) \leq 0 .
$$

(H2) There exists $\alpha>0$ such that the roots of the equation $\operatorname{det}\left(s I-\sum_{l=0}^{L} A_{l} e^{-h_{l} s}\right)=0$ have real part smaller than $-\alpha$.

Assume that there exists a diagonal matrix $\eta=\operatorname{diag}\left\{\eta_{i}\right\}$ such that the following Hypothesis is fulfilled

(H3) There exist $\gamma: \mathbb{R}^{+} \rightarrow \mathbb{R}$ with $\lim _{z \rightarrow 0} \gamma(z)=0$ and $D_{j}=\operatorname{diag}\left\{D_{j, i}\right\}, j \in\{1,2,3\}$, such that

$$
\begin{aligned}
& \text { for almost any } t \in \mathbb{R}^{+}, \forall y \in \mathcal{O}, \forall i \in\{1, \ldots, p\} \\
& \qquad \eta_{i}\left(\int_{0}^{y_{i}} \frac{\partial \psi_{i}}{\partial t}(t, z) d z-D_{1, i} y_{i}^{2}-D_{2, i} y_{i} \psi_{i}\left(t, y_{i}\right)-D_{3, i} \psi_{i}\left(t, y_{i}\right)^{2}\right) \leq\|y\|^{2} \gamma(\|y\|) .
\end{aligned}
$$

and such that the transfer function matrix

$$
I-\eta K D_{3}+\left(I+\eta\left(s I+D_{2}\right)\right) K H(s)-H^{*}(s) \sup \left\{\eta D_{1} ; \frac{|\eta|-\eta}{2} K\left(D_{2}+K D_{3}\right)\right\} K H(s) \quad \text { is } S P R .
$$

Then, the origin of system (1) is uniformly locally asymptotically stable.

Moreover, if $\gamma \equiv 0$ and $\mathcal{O}=\mathbb{R}^{p}$, then the origin of system (1) is uniformly globally asymptotically stable.

A proof of Theorem 1 is presented in Section 5. It essentially follows the approach of [26, 12], with adequate improvements.

Circle criterion is found as a particular case for $\eta=0$, and Popov criterion when $\frac{\partial \psi}{\partial t}=0$ (taking $D_{j}=0,(7)$ reduces to $(3)$ ).

Remark that if Hypothesis (H3) holds for a certain $\eta$, then it holds (with the same $D_{j}$ and $\gamma$ ) for any $\eta^{\prime}$ such that $\eta \eta^{\prime}>0$ : only the sign of the $\eta_{i}$ 's intervenes. In practice, one first determinates the sign of the $\eta_{i}$ 's which lead to an estimate like (6). Under these sign constraints on the $\eta_{i}$ 's, one then verifies (7). The matrices $D_{j}, j=1,2,3$, may depend upon $\operatorname{sgn} \eta$.

An important case where Hypothesis (H3) is fulfilled leads to the following result.

Corollary 2. Assume that there exists a convex open neighborhood $\mathcal{O}$ of 0 in $\mathbb{R}^{p}$ for which (H0), (H1), (H2) hold. Assume that there exists a diagonal matrix $\eta$ such that the following Hypothesis is fulfilled

(H3') There exist $\gamma: \mathbb{R}^{+} \rightarrow \mathbb{R}$ with $\lim _{z \rightarrow 0} \gamma(z)=0$ and $\Delta=\operatorname{diag}\left\{\Delta_{i}\right\}, \Delta_{i}: \mathbb{R}^{+} \rightarrow \mathbb{R}$, such that,

$$
\text { for almost any } t \in \mathbb{R}^{+}, \forall y \in \mathcal{O}, \quad y^{T} \eta\left(\frac{\partial \psi}{\partial t}(t, y)-\Delta(t) y\right) \leq\|y\|^{2} \gamma(\|y\|) \text {. }
$$

and such that the transfer function matrix

$$
I+(I+\eta s) K H(s)-\frac{1}{2} H^{*}(s) K^{\frac{1}{2}} \sup _{t \geq 0} \operatorname{ess}\{\eta \Delta(t)\} K^{\frac{1}{2}} H(s) \quad \text { is } S P R .
$$

Then, the origin of system (1) is uniformly locally asymptotically stable.

Moreover, if $\gamma \equiv 0$ and $\mathcal{O}=\mathbb{R}^{p}$, then the origin of system (1) is uniformly globally asymptotically stable. 
One checks easily that (H3') implies (H3) with $D_{2}=D_{3}=0$ and

$$
D_{1, i}=\frac{1}{2} \sup _{t \geq 0} \operatorname{ess}\left\{\Delta_{i}(t)\right\} \text { if } \eta_{i} \geq 0, D_{1, i}=\frac{1}{2} \inf _{t \geq 0} \operatorname{ess}_{i}\left\{\Delta_{i}(t)\right\} \text { if } \eta_{i} \leq 0, D_{2}=D_{3}=0 .
$$

Indeed, when (8) holds,

$$
\inf _{t \geq 0} \operatorname{ess} \Delta(t) \leq 0 \leq \sup _{t \geq 0} \operatorname{ess} \Delta(t)
$$

otherwise sector condition (5) would be violated, so

$$
\eta D_{1}=\sup _{t \geq 0} \operatorname{ess}\{\eta \Delta(t)\}=|\eta|_{+} \sup _{t \geq 0} \operatorname{ess}\{\Delta(t)\}+|\eta|_{-} \inf _{t \geq 0} \operatorname{ess}\{\Delta(t)\} \geq 0 .
$$

The additional, quadratic, term in (9) is then nonpositive, indicating clearly that the criterion is more restrictive than Popov conditions (compare with (3)) for the systems with nonautonomous nonlinearities.

Condition (H3') is in general a "local" sector condition. It is fulfilled in two important cases. When $\gamma \equiv 0$ (1st case),$(8)$ writes:

$$
\text { for almost any } t \in \mathbb{R}^{+}, \forall y \in \mathcal{O}, \quad y^{T} \eta\left(\frac{\partial \psi}{\partial t}(t, y)-\Delta(t) y\right) \leq 0
$$

that is:

$$
\forall i \in\{1, \ldots, p\}, \text { for almost any } t \in \mathbb{R}^{+}, \forall y \in \mathcal{O},\left\{\begin{array}{ll}
\frac{1}{y_{i}} \frac{\partial \psi_{i}}{\partial t}\left(t, y_{i}\right) \leq \Delta_{i}(t) t-\text { a.e. } & \text { if } \eta_{i} \geq 0 \\
\frac{1}{y_{i}} \frac{\partial \psi_{i}}{\partial t}\left(t, y_{i}\right) \geq \Delta_{i}(t) t-\text { a.e. } & \text { if } \eta_{i} \leq 0
\end{array}\right. \text {. }
$$

This hence defines a ("global") sector condition on the map $y \mapsto \frac{\partial \psi}{\partial t}(t, y)$. When the inequality in (8) is replaced by an equality ( 2 nd case), then,

$$
\forall i \in\{1, \ldots, p\}, \text { for almost any } t \in \mathbb{R}^{+}, \forall y \in \mathcal{O},\left|\frac{\partial \psi_{i}}{\partial t}\left(t, y_{i}\right)-\Delta_{i}(t) y_{i}\right|=\left|y_{i}\right| \gamma\left(\left|y_{i}\right|\right),
$$

which means that $\frac{\partial^{2} \psi_{i}}{\partial y_{i} \partial t}(t, 0)$ exists almost everywhere and is equal to $\Delta_{i}(t)$. Condition (8) is a generalization of $(6)$, whose idea is borrowed from [32, 14].

Remark that $\psi$ does not have to be continuous wrt $y$, except in 0 , and the same is true for $\frac{\partial \psi}{\partial t}$. In the conditions of application of Theorem 1, there exist functions $k_{i}\left(t, y_{i}\right)$ such that $\psi_{i}\left(t, y_{i}\right)=k_{i}\left(t, y_{i}\right) y_{i}$, $i=1, \ldots, p$, and it may be fruitful to express the results in terms of the $k_{i}$. As an example, (H1) requires that $0 \leq k_{i}\left(t, y_{i}\right) \leq K_{i}$. Also, (8) expresses that

$$
\forall i \in\{1, \ldots, p\}, \text { for almost any } t \in \mathbb{R}^{+}, \forall y \in \mathcal{O}, \eta_{i}\left(\frac{\partial k_{i}}{\partial t}\left(t, y_{i}\right)-\Delta_{i}(t)\right) \leq \gamma\left(\left\|y_{i}\right\|\right),
$$

and $\frac{\partial^{2} \psi_{i}}{\partial y_{i} \partial t}(t, 0)=\frac{\partial k_{i}}{\partial t}(t, 0)$ when the 2 nd derivative exists.

When the solutions of (1) are continuous wrt the initial conditions, one may consider the essential suprema [34] of $\eta \Delta(t)$ on $\left[t_{0},+\infty\right)$ for any $t_{0} \geq 0$, instead of $[0,+\infty)$. Indeed, due to the strict inequality involved, one may even use the upper limit [34] of these expressions when $t_{0} \rightarrow+\infty$.

For a scalar system, $p=1$, and condition (7) is equivalent to

$$
\begin{aligned}
\exists \eta \in \mathbb{R}, \forall \omega \in \mathbb{R}, \quad \frac{1}{K}+\operatorname{Re} H(j \omega)-\eta\left(D_{3}-D_{2} \operatorname{Re} H(j \omega)+\omega \operatorname{Im} H(j \omega)\right) \\
+\sup \left\{\eta D_{1} ; \frac{|\eta|-\eta}{2} K\left(D_{2}+K D_{3}\right)\right\}|H(j \omega)|^{2} \geq 0 .
\end{aligned}
$$

This may be interpreted graphically, as in [5]: 
If, apart from the regularity, sector and stability conditions $(\mathrm{H} 0),(\mathrm{H} 1),(\mathrm{H} 2),(\mathrm{H} 3)$, there exists a line of slope $1 / \eta$ passing through the point $\left(-\frac{1}{K}, 0\right)$ and lying to the left of the locus $\left(\operatorname{Re} H(j \omega), D_{3}-D_{2} \operatorname{Re} H(j \omega)+\omega \operatorname{Im} H(j \omega)+\operatorname{sgn} \eta \cdot \sup \left\{\operatorname{sgn} \eta \cdot D_{1} ; \frac{1-\operatorname{sgn} \eta}{2} K\left(D_{2}+K D_{3}\right)\right\}\right.$ $\left.|H(j \omega)|^{2}\right)$ without intersecting it, then the uniform local stability property holds. If $\gamma \equiv 0$ and $\mathcal{O}=\mathbb{R}^{p}$, then the uniform global stability property holds.

An interesting problem is, given $K$, to determinate the largest incertitude on $\frac{\partial \psi}{\partial t}$ allowed by Theorem 1 , for example under condition (H3'). In this case, the previous graphical criterion can hardly be used, as the ordinate changes with the $D_{j}$. To overcome this drawback, condition (7) should rather be seen as a geometrical condition in the 3-dimensional space $(\operatorname{Re} H(j \omega), \operatorname{Im} H(j \omega), \omega \operatorname{Im} H(j \omega))$ obtained as the product of Nyquist and Popov planes, a condition not easy to interpret. We present in the sequel a weaker but simpler condition, located in the Popov plane.

Denoting the $\mathcal{H}_{\infty}$-norm of $H$ by $\|H(s)\|_{\infty} \stackrel{\text { def }}{=} \sup \{\|H(s)\|: \operatorname{Re} s>0\}$ (when $H$ is stable and proper, this is equal to $\sup \{\|H(j \omega)\|: \omega \in \mathbb{R}\}$ ), one deduces easily the following result.

Theorem 3 (A weaker frequency criterion). Assume that there exists a convex open neighborhood $\mathcal{O}$ of 0 in $\mathbb{R}^{p}$ for which Hypotheses (H0), (H1), (H2) hold. If there exists a diagonal matrix $\eta$ such that (H3) is fulfilled and such that the transfer function matrix

$$
I-\eta D_{3}+\left(I+\eta\left(s I+D_{2}\right)\right) K H(s)-K \sup \left\{\eta D_{1} ; \frac{|\eta|-\eta}{2} K\left(D_{2}+K D_{3}\right) ; 0\right\}\|H(s)\|_{\infty}^{2} \quad \text { is } S P R,
$$

then the conclusions of Theorem 1 hold.

As an example let us examine the case of a scalar system fulfilling (H3'), the general case (H3) is similar. Formula (11) is equivalent to (12a) (resp. (12b)) for $\eta \geq 0$ (resp. $\eta \leq 0$ ), where

$$
\begin{aligned}
& \exists \eta \geq 0, \forall \omega \in \mathbb{R}, \frac{1}{K}+\operatorname{Re} H(j \omega)-\eta\left(\omega \operatorname{Im} H(j \omega)+\frac{1}{2}\|H(s)\|_{\infty}^{2} \sup _{t \geq 0}^{\operatorname{ess}}\{\Delta(t)\}\right) \geq 0, \\
& \exists \eta \leq 0, \forall \omega \in \mathbb{R}, \frac{1}{K}+\operatorname{Re} H(j \omega)-\eta\left(\omega \operatorname{Im} H(j \omega)+\frac{1}{2}\|H(s)\|_{\infty}^{2} \underset{t \geq 0}{\inf } \operatorname{ess}\{\Delta(t)\}\right) \geq 0,
\end{aligned}
$$

and this has a clear interpretation:

If, apart from the regularity, sector and stability conditions (H0), (H1), (H2), (H3') with $\eta \geq 0$ (resp. $\eta \leq 0)$, a line of slope $1 / \eta$ passing through the point $\left(-\frac{1}{K}, 0\right)$ lies above (resp. below) the Popov locus and may be translated vertically towards the locus by a distance

$$
\frac{1}{2}\|H(s)\|_{\infty}^{2} \sup _{t \geq 0} \operatorname{ess}\{(t)\} \quad\left(\text { resp. }-\frac{1}{2}\|H(s)\|_{\infty}^{2} \underset{t \geq 0}{\inf \operatorname{ess}}\{\Delta(t)\}\right)
$$

without intersecting it, then the uniform local stability property holds. If $\gamma \equiv 0$ and $\mathcal{O}=\mathbb{R}^{p}$, then the uniform global stability property holds.

This is illustrated in Figure 2: in the left (resp. right) Popov diagram, (12a) (resp. (12b)) holds if

$$
\sup _{t \geq 0} \operatorname{ess} \Delta(t)<\frac{2 d}{\|H(s)\|_{\infty}^{2}} \quad\left(\text { resp. inf } \operatorname{ess}_{t \geq 0} \Delta(t)>-\frac{2 d}{\|H(s)\|_{\infty}^{2}}\right)
$$

As an example, (13) holds if (1st case)

$$
\forall y \in \mathcal{O}, \quad \frac{1}{y} \frac{\partial \psi}{\partial t}(t, y)<\frac{2 d}{\|H(s)\|_{\infty}^{2}} t-\text { a.e. } \quad\left(\text { resp. } \frac{1}{y} \frac{\partial \psi}{\partial t}(t, y)>-\frac{2 d}{\|H(s)\|_{\infty}^{2}} t-\text { a.e. }\right)
$$

or if (2nd case)

$$
\frac{\partial^{2} \psi}{\partial y \partial t}(t, 0)<\frac{2 d}{\|H(s)\|_{\infty}^{2}} t-\text { a.e. } \quad\left(\text { resp. } \frac{\partial^{2} \psi}{\partial y \partial t}(t, 0)>-\frac{2 d}{\|H(s)\|_{\infty}^{2}} t-\text { a.e. }\right) .
$$



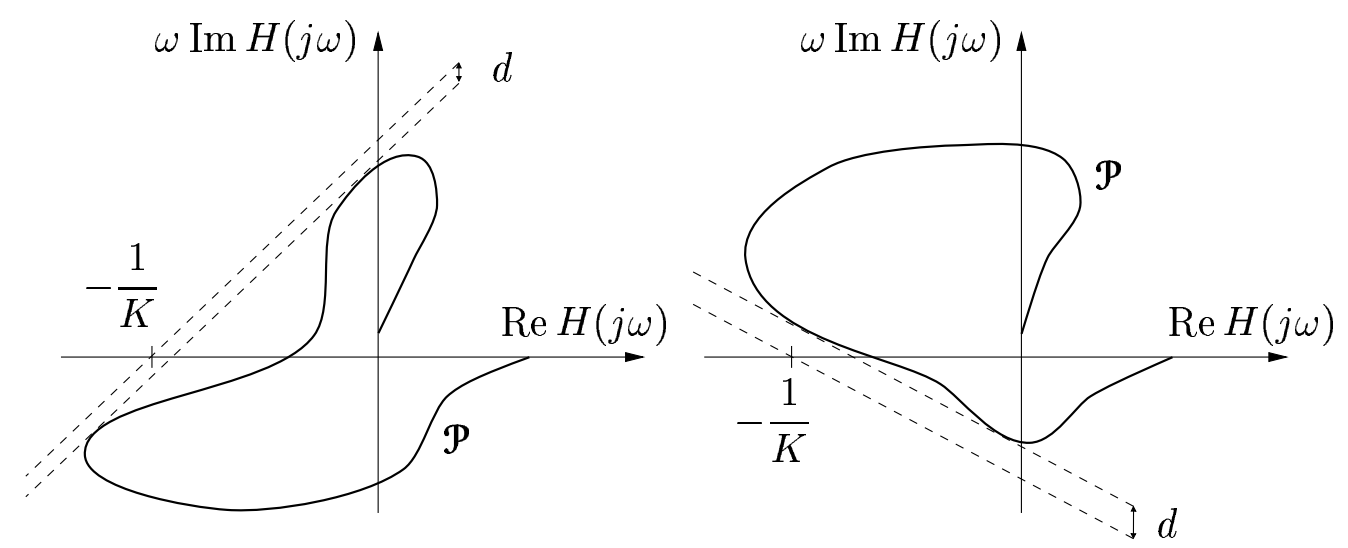

Figure 2: Graphical stability criterion in the Popov plane

Both cases are fulfilled when

$$
\text { for almost any } t \in \mathbb{R}^{+}, \frac{\partial \psi}{\partial t}(t, y) \text { is linear wrt } y \in \mathcal{O} \text {. }
$$

In the configurations shown in Figure 2, the quantity $d$ involved is indeed the least $z>0$ such that one of the points $(-1 / K, \pm z)$ belongs to the convex hull of the Popov locus $\mathcal{P}$. To show this, remark that, e.g. for the right diagram,

$$
d=-\inf _{\eta \in \mathbb{R}} \sup _{\omega \in \mathbb{R}}\left(\eta\left(\operatorname{Re} H(j \omega)+\frac{1}{K}\right)-\omega \operatorname{Im} H(j \omega)\right) .
$$

Now, $\sup _{\omega \in \mathbb{R}} \eta(\operatorname{Re} H(j \omega)+1 / K)-\omega \operatorname{Im} H(j \omega)$ may be seen as the value of the support function of the set $\mathcal{P}_{+1} / K$ applied to the vector $(\eta,-1)$ [33]. One may hence replace the set $\mathcal{P}$ by its convex hull conv $\mathcal{P}$, and then reverse the order of inf and sup. One gets:

$$
d=-\sup \left\{\inf _{\eta \in \mathbb{R}} \eta\left(z_{1}+1 / K\right)-z_{2}:\left(z_{1}, z_{2}\right) \in \operatorname{conv} \mathfrak{P}\right\}=\inf \left\{z_{2}:\left(-1 / K, z_{2}\right) \in \operatorname{conv} \mathfrak{P}\right\}
$$

\section{Computation issues}

It turns out that for rational systems, frequency condition (7) may be checked easily:

Proposition 4 (LMI condition for rational systems). Let $H$ be a rational strictly proper Hurwitz transfer function matrix, $(A, B, C)$ a minimal realization. The following $L M I$ is feasible:

$$
\begin{aligned}
& P>0, \quad \eta^{\prime} \stackrel{\text { def }}{=} \operatorname{diag}\left\{\eta_{i}^{\prime}\right\}, \\
& A^{T} P+P A \\
& \left(\begin{array}{cc}
-(P-W) B+C^{T} K+A^{T} C^{T} K \eta^{\prime}+C^{T} K \eta^{\prime} D_{2} \\
-B^{T}(P-W)+K C+\eta^{\prime} K C A+D_{2} \eta^{\prime} K C & -2 I-\eta^{\prime} K C B-B^{T} C^{T} K \eta^{\prime}+2 \eta^{\prime} K D_{3}
\end{array}\right)<0,
\end{aligned}
$$

where $W=W^{T}$ is defined by

$$
A^{T} W+W A=2 C^{T} K \sup \left\{\eta^{\prime} D_{1} ; \frac{\left|\eta^{\prime}\right|-\eta^{\prime}}{2} K\left(D_{2}+K D_{3}\right)\right\} C
$$

if and only if there exists a diagonal matrix $\eta$ such that condition (7) holds and $\eta \eta^{\prime} \geq 0$. 
Sketch of the proof. The idea is to apply Kalman-Yakubovich-Popov (KYP) Lemma. The verification of the observability condition (needed to apply KYP Lemma) necessitates special care, see [6] for a complete proof.

In order to apply Theorem 1, it then suffices to achieve approximation by rational transfers, see e.g. [23, 13] for the techniques of approximation. The following result states this properly.

Proposition 5 (Transfer approximation). Let $H, H_{n}$ be strictly proper Hurwitz transfer function matrices. Let $\eta$ be a diagonal matrix.

Suppose that there exists $\varepsilon \in(0,1)$ such that

$$
\begin{aligned}
&(1-\varepsilon) I-\eta K D_{3}+\left(I+\eta\left(s I+D_{2}\right)\right) K H_{n}(s) \\
&-H_{n}^{*}(s) K \sup \left\{\eta D_{1} ; \frac{|\eta|-\eta}{2} K\left(D_{2}+K D_{3}\right)\right\} H_{n}(s) \quad \text { is SPR, } \\
& \varepsilon I+\left(I+\eta\left(s I+D_{2}\right)-2 H^{*}(s) \sup \left\{\eta D_{1} ; \frac{|\eta|-\eta}{2} K\left(D_{2}+K D_{3}\right)\right\}\right) K\left(H-H_{n}\right)(s) \\
&+\left(H-H_{n}\right)^{*}(s) \sup \left\{\eta D_{1} ; \frac{|\eta|-\eta}{2} K\left(D_{2}+K D_{3}\right)\right\} K\left(H-H_{n}\right)(s) \quad \text { is SPR. }
\end{aligned}
$$

Then condition (7) holds.

Conversely, suppose that there exists $\varepsilon>0$ such that

$$
\begin{aligned}
& (1+\varepsilon) I-\eta K D_{3}+\left(I+\eta\left(s I+D_{2}\right)\right) K H_{n}(s) \\
& -H_{n}^{*}(s) K \sup \left\{\eta D_{1} ; \frac{|\eta|-\eta}{2} K\left(D_{2}+K D_{3}\right)\right\} H_{n}(s) \quad \text { is NOT } S P R, \\
& \varepsilon I-\left(I+\eta\left(s I+D_{2}\right)-2 H^{*}(s) \sup \left\{\eta D_{1} ; \frac{|\eta|-\eta}{2} K\left(D_{2}+K D_{3}\right)\right\}\right) K\left(H-H_{n}\right)(s) \\
& -\left(H-H_{n}\right)^{*}(s) \sup \left\{\eta D_{1} ; \frac{|\eta|-\eta}{2} K\left(D_{2}+K D_{3}\right)\right\} K\left(H-H_{n}\right)(s) \quad \text { is SPR. }
\end{aligned}
$$

Then condition (7) does not hold.

The proof is left to the reader. Assumption (16a) (resp. assumption (17a)) is slightly stronger than the assumption needed to apply Theorem 1 to $H_{n}$ (resp. slightly weaker than the negation of this assumption). Assumptions (16b) or (17b) are fulfilled e.g. when $\left\|H-H_{n}\right\|_{\infty}$ is small enough.

\section{An example: computation of stability margin for a 4th order system with delay}

One shall consider the following system:

$$
16 \frac{d^{4} y}{d t^{4}}+32 \frac{d^{3} y}{d t^{3}}+24 \frac{d^{2} y}{d t^{2}}+8 \frac{d y}{d t}+y=-\psi(t, y(t-1)),
$$

corresponding to the previously studied framework with the transfer function

$$
H(s)=\frac{e^{-s}}{(1+2 s)^{4}}
$$

which may easily be realized as in (1). 
One supposes that $\psi(t, 0) \equiv 0$ and that there exists a constant $K$ such that

$$
y \neq 0 \Rightarrow 0 \leq \frac{\psi(t, y)}{y} \leq K .
$$

One verifies either graphically (see Figure 3 ) or by computations, that asymptotic stability of system (18) cannot be guaranteed if

$$
K \geq \frac{1}{0.3613} \simeq 2.768
$$

This corresponds to the smallest positive value of $K$ for which the equation $1+K H(s)=0$ has some purely imaginary roots.

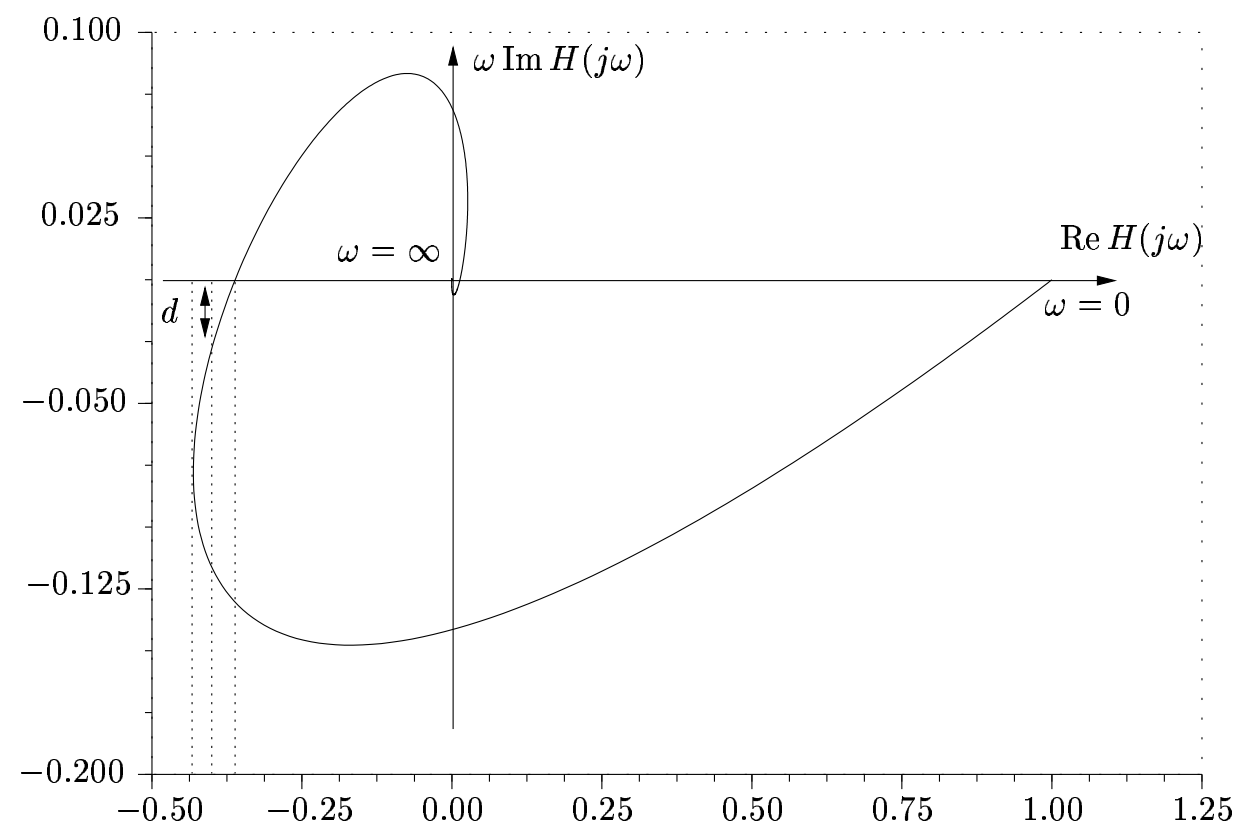

Figure 3: Popov locus of system (18)

Application of circle criterion provides stability if

$$
K<\frac{1}{0.4310} \simeq 2.320
$$

On the other hand, Popov criterion guarantees stability if $\psi(t, y)=\psi(y)$ and

$$
K<2.768 \text {. }
$$

In the remaining of the section, one studies the stability of (18) for time-varying nonlinearities fulfilling the sector condition with

$$
K=1 / 0.4=2.5 \in[2.320,2.768] .
$$

More precisely, let us assume that condition (H3') holds; one is looking for restrictions on $\Delta(t)$ permitting to ensure stability. The results previously exposed permit to compute measures of the stability robustness. 
In the sequel, we provide lower estimates of the largest number $\delta$ such that system (18) is absolute stable whenever

$$
\sup _{t \geq 0} \operatorname{ess} \Delta(t) \leq \delta
$$

All computations to be presented have been achieved using the Scilab package LMITOOL $^{1}$.

Using the ideas of Section 3, one approximates the delay following [23], and considers the sequence of approximants:

$$
H_{n}(s)=\left(\frac{1-\frac{s}{2 n}}{1+\frac{s}{2 n}}\right)^{n} \frac{1}{(1+2 s)^{4}}
$$

For each value of $n$, using Proposition 4 and proceeding by dichotomy, one may solve the LMI (14), (15). This process provides a lower estimate of $\delta$, denoted $\delta_{1}$. The results are presented in Table 1 . The exact

\begin{tabular}{|c||c|} 
Order of approximation & Successive estimates of $\delta_{1}$ \\
\hline$n=1$ & 0.3444 \\
$n=2$ & 0.3365 \\
$n=3$ & 0.3350 \\
$n=4$ & 0.3345 \\
$n=5$ & 0.3342 \\
\hline$n=+\infty$ & 0.3338 \\
\hline
\end{tabular}

Table 1: Computation of the robustness measure $\delta_{1}$

value of $\delta_{1}$ may be computed numerically for scalar systems, as (9) is fulfilled for a positive value of $\eta$ if and only if

$$
\sup _{t \geq 0} \operatorname{ess} \Delta(t)<\sup _{\eta>0} \inf _{\omega \in \mathbb{R}} \frac{2}{|H(j \omega)|^{2}}\left(\frac{1}{\eta}\left(\frac{1}{K}+\operatorname{Re} H(j \omega)\right)-\omega \operatorname{Im} H(j \omega)\right) .
$$

The optimal value of $\eta$ is $\eta_{\mathrm{opt}} \simeq 1.473$. In conclusion, condition (9) is fulfilled if

$$
\sup _{t \geq 0} \operatorname{ess} \Delta(t) \leq \delta_{1} \stackrel{\text { def }}{=} 0.3338
$$

The corresponding graphical interpretation may be read on Figure 4.

One now shows how to compute a bound on $\delta$ with the help of Theorem 3. First the quantity $d$ is evaluated, either graphically, or using a solver of algebraic equations. One gets:

$$
d \simeq 0.03325 .
$$

One then computes $\|H\|_{\infty}$. This step may also be achieved by use of rational approximations. One obtains here:

$$
\|H\|_{\infty}=1
$$

Condition (11) is hence fulfilled if

$$
\sup _{t \geq 0} \operatorname{ess} \Delta(t) \leq \delta_{2} \stackrel{\text { def }}{=} \frac{2 d}{\|H\|_{\infty}^{2}} \simeq 0.06650
$$

\footnotetext{
${ }^{1}$ Scilab is a free software developed by INRIA, which is distributed with all its source code. For the distribution and details, see Scilab's homepage on the web at the address http://www-rocq.inria.fr/scilab/
} 


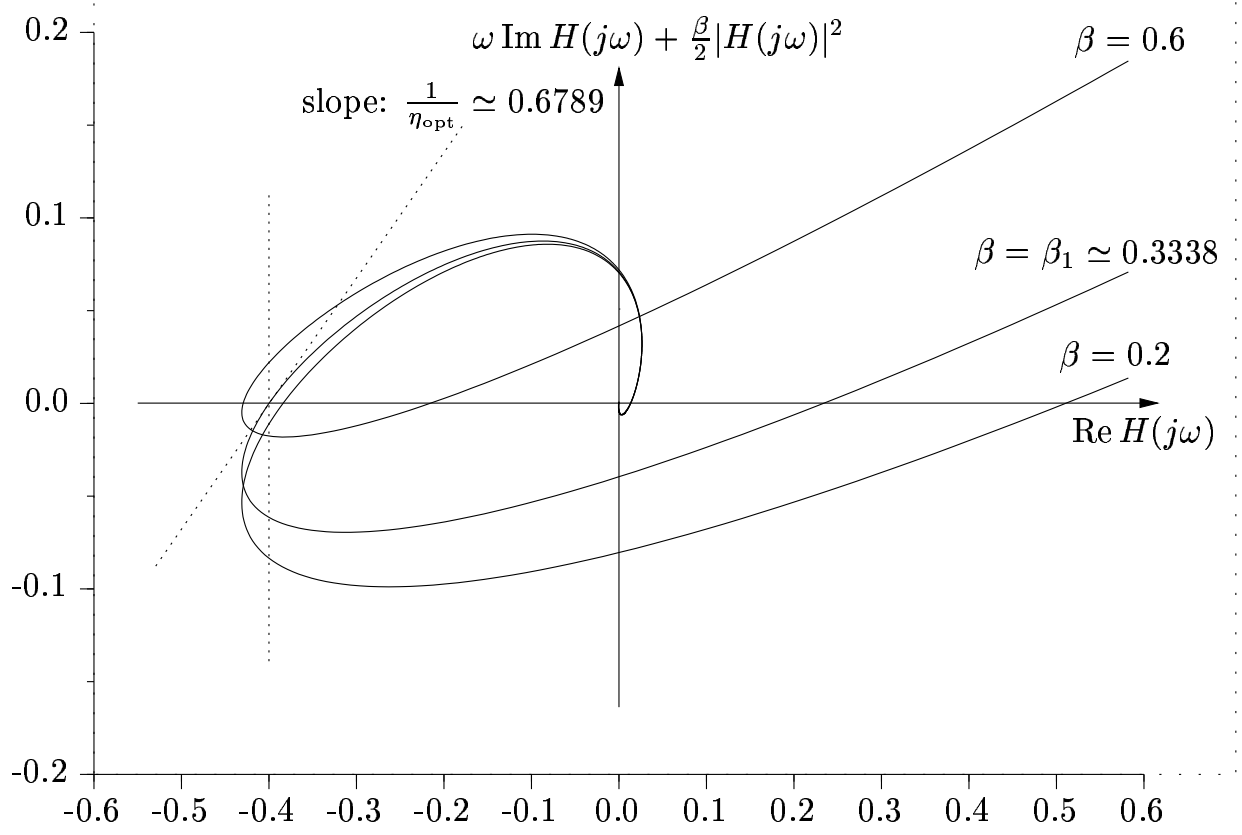

Figure 4: Graphical interpretation of Theorem 1 for system (18)

One verifies that the ordering

$$
\delta_{1}>\delta_{2}
$$

is consistent with the increasing conservativeness of the criteria.

Let us give a sample of the results that may be obtained. Let $\mathcal{O}$ be a convex open neighborhood of 0 in $\mathbb{R}$, such that

- There exists $L \in L_{l o c}^{1}(\mathcal{O})$ such that, for all $t, t^{\prime} \in \mathbb{R}^{+}$, for all $y \in \mathcal{O},\left|\psi(t, y)-\psi\left(t^{\prime}, y\right)\right| \leq L(y)\left|t-t^{\prime}\right|$ (condition (H0)).

- For all $t \in \mathbb{R}^{+}$, for all $y \in \mathcal{O} \backslash\{0\}, 0 \leq \frac{\psi(t, y)}{y} \leq 2.5$, and $\psi(t, 0) \equiv 0$ (condition (H1)).

Assume that there exist global solutions to system (18). The origin of system (18) is uniformly locally stable if

$$
\forall y \in \mathcal{O} \backslash\{0\}, \quad \frac{1}{y} \frac{\partial \psi}{\partial t}(t, y) \leq \delta_{1}+\gamma(|y|) t-\text { a.e. , with } \lim _{|y| \rightarrow 0} \gamma(|y|)=0,
$$

for example if $\frac{\partial^{2} \psi}{\partial y \partial t}(t, 0)$ exists $t$-a.e. and verifies:

$$
\frac{\partial^{2} \psi}{\partial y \partial t}(t, 0) \leq \delta_{1} t-\text { a.e. }
$$

The origin of system (18) is uniformly globally stable if $\mathcal{O}=\mathbb{R}$ and

$$
\forall y \in \mathbb{R} \backslash\{0\}, \quad \frac{1}{y} \frac{\partial \psi}{\partial t}(t, y) \leq \delta_{1} t \text { - a.e. }
$$




\section{Proof of Theorem 1}

One assumes, without loss of generality, that $\gamma$ is nonnegative, nondecreasing.

- Let us first suppose that $\eta \geq 0$. The demonstration begins as in $[26,12]$.

Let $T>0$. Let us define $\psi_{T}, x_{T}, y_{T}$ as follows

$$
\begin{gathered}
\psi_{T}(t)= \begin{cases}\psi(t, y(t)) & \text { if } 0 \leq t \leq T \\
0 & \text { if }-h \leq t<0 \text { or } t>T\end{cases} \\
\dot{x}_{T}=\sum_{l=0}^{L} A_{l} x_{T}\left(t-h_{l}\right)-B \psi_{T}, \quad y_{T}=\sum_{l=0}^{L} C_{l} x_{T}\left(t-h_{l}\right),\left.\quad x_{T}\right|_{[-h, 0]}=0 .
\end{gathered}
$$

By linearity, we have

$$
\dot{x}-\dot{x}_{T}=\sum_{l=0}^{L} A_{l}\left(x\left(t-h_{l}\right)-x_{T}\left(t-h_{l}\right)\right) \quad \text { for } t \in[0, T],\left.\quad\left(x-x_{T}\right)\right|_{[-h, 0]}=\phi,
$$

We shall denote in the sequel by $c_{j}, j=1,2, \ldots$, various positive constants, independent of $\phi$ and $T$. From Hypothesis (H2), we deduce that there exists $\alpha^{\prime} \in(0, \alpha)$, independent of $\phi$ and $T$, such that

$$
\left\{\begin{array}{l}
\forall t \in[-h, T], \quad\left\|x(t)-x_{T}(t)\right\| \leq c_{1} e^{-\alpha^{\prime} t}\|\phi\|_{\mathcal{C}([-h, 0])} \\
\forall t \geq T, \quad\left\|x_{T}(t)\right\| \leq c_{1} e^{-\alpha^{\prime}(t-T)}\left\|x_{T}(T+\cdot)\right\|_{\mathcal{C}([-h, 0])}
\end{array}\right.
$$

This implies, using triangular inequality:

$$
\forall t \in[0, T],\|y(t)\| \leq c_{2} e^{-\alpha^{\prime} t}\|\phi\|_{\mathcal{C}([-h, 0])}+\left\|y_{T}(t)\right\| .
$$

From (H1) and the fact that $\eta, K \geq 0$, one gets that, if $y(t) \in \mathcal{O}, t \in[0, T]$, then

$$
\forall i \in\{1, \ldots, p\}, \quad \int_{0}^{T}\left(K_{i} y_{i}(t)-\psi_{i}\left(t, y_{i}(t)\right)\right) \psi_{i}\left(t, y_{i}(t)\right) d t+\eta_{i} K_{i} \int_{0}^{y_{i}(T)} \psi_{i}(T, z) d z \geq 0 .
$$

On the other hand, the map

$$
t \mapsto \int_{0}^{y_{i}(t)} \psi_{i}(t, z) d z
$$

is absolutely continuous, due to (H0) and (H1), and the assumed absolute continuity of the solution wrt time. Indeed, $y(t), y\left(t^{\prime}\right) \in \mathcal{O}$ implies that, $\forall i \in\{1, \ldots, p\}$,

$$
\left|\int_{0}^{y_{i}(t)} \psi_{i}(t, z) d z-\int_{0}^{y_{i}\left(t^{\prime}\right)} \psi_{i}\left(t^{\prime}, z\right) d z\right| \leq\left|t-t^{\prime}\right| \int_{0}^{y_{i}(t)} L_{i}(z) d z+K_{i} \max \left\{\left|y_{i}(t)\right|,\left|y_{i}\left(t^{\prime}\right)\right|\right\}\left|y_{i}(t)-y_{i}\left(t^{\prime}\right)\right|,
$$

where $L_{i}$ is the Lispchitz constant of $\psi$, defined by Hypothesis (H1). One may hence write

$$
\begin{aligned}
\int_{0}^{y_{i}(T)} \psi_{i}(T, z) d z & =\int_{0}^{T} \frac{d}{d t}\left[\int_{0}^{y_{i}(t)} \psi_{i}(t, z) d z\right] d t+\int_{0}^{y_{i}(0)} \psi_{i}(0, z) d z \\
& =\int_{0}^{T}\left(\dot{y}_{i}(t) \psi_{T, i}(t)+\int_{0}^{y_{i}(t)} \frac{\partial \psi_{i}}{\partial t}(t, z) d z\right) d t+\int_{0}^{y_{i}(0)} \psi_{i}(0, z) d z
\end{aligned}
$$

Now, if $y(t) \in \mathcal{O}$ for any $t \in[0, T]$, one has, using (H3),

$$
\begin{aligned}
& \eta_{i} \int_{0}^{T} \int_{0}^{y_{i}(t)} \frac{\partial \psi_{i}}{\partial t}(t, z) d z d t \\
& \leq \int_{0}^{T}\left(\eta_{i}\left(D_{1, i} y_{i}^{2}(t)+D_{2, i} y_{i}(t) \psi_{T, i}(t)+D_{3, i} \psi_{T, i}(t)^{2}\right)+\gamma\left(\left|y_{i}(t)\right|\right) y_{i}^{2}(t)\right) d t
\end{aligned}
$$


because $\gamma$ is nonnegative and nondecreasing, and $\mathcal{O}$ is convex. One hence deduces from (24)

$$
\begin{aligned}
\int_{0}^{T}( & \left.K_{i}\left(y_{i}(t)-y_{T, i}(t)\right) \psi_{T, i}(t)+K_{i} \gamma\left(\left|y_{i}(t)\right|\right) y_{i}^{2}(t)\right) d t+\eta_{i} K_{i} \int_{0}^{y_{i}(0)} \psi_{i}(0, z) d z \\
\geq & -\int_{0}^{T}\left(\left(K_{i} y_{T, i}(t)-\psi_{T, i}(t)\right) \psi_{T, i}(t)\right. \\
& \left.+\eta_{i} K_{i}\left(\dot{y}_{i}(t) \psi_{T, i}(t)+D_{1, i} y_{i}^{2}(t)+D_{2, i} y_{i}(t) \psi_{T, i}(t)+D_{3, i} \psi_{T, i}(t)^{2}\right)\right) d t \\
= & -\int_{0}^{T}\left(\left(K_{i} y_{T, i}(t)-\psi_{T, i}(t)\right) \psi_{T, i}(t)\right. \\
& \left.+\eta_{i} K_{i}\left(\dot{y}_{T, i}(t) \psi_{T, i}(t)+D_{1, i} y_{T, i}^{2}(t)+D_{2, i} y_{T, i}(t) \psi_{T, i}(t)+D_{3, i} \psi_{T, i}(t)^{2}\right)\right) d t \\
& +\eta_{i} K_{i} \int_{0}^{T}\left(\left(\dot{y}_{T, i}(t)-\dot{y}_{i}(t)\right) \psi_{T, i}(t)+D_{1, i}\left(y_{T, i}^{2}(t)-y_{i}^{2}(t)\right)+D_{2, i}\left(y_{T, i}(t)-y_{i}(t)\right) \psi_{T, i}(t)\right) d t \\
\geq & -\int_{0}^{+\infty}\left(\left(K_{i} y_{T, i}(t)-\psi_{T, i}(t)\right) \psi_{T, i}(t)+\left|\eta_{i} D_{1, i}\right|+K_{i} y_{T, i}^{2}(t)\right. \\
& \left.+\eta_{i} K_{i}\left(\dot{y}_{T, i}(t) \psi_{T, i}(t)+D_{2, i} y_{T, i}(t) \psi_{T, i}(t)+D_{3, i} \psi_{T, i}(t)^{2}\right)\right) d t \\
& +\eta_{i} K_{i} \int_{0}^{T}\left(\left(\dot{y}_{T, i}(t)-\dot{y}_{i}(t)\right) \psi_{T, i}(t)+D_{1, i}\left(y_{T, i}^{2}(t)-y_{i}^{2}(t)\right)+D_{2, i}\left(y_{T, i}(t)-y_{i}(t)\right) \psi_{T, i}(t)\right) d t
\end{aligned}
$$

as $K \geq 0, \psi_{T}(t)=0$ for $t \geq T$, and because $y_{T, i}^{2}$ is summable over $\mathbb{R}^{+}$, due to (22). Denoting $\tilde{y}_{T}, \tilde{\psi}_{T}$ the Fourier transform of $y_{T}, \psi_{T}$, the previous indefinite integral is proved to be equal to

$$
\begin{array}{r}
\frac{1}{2 \pi} \int_{-\infty}^{+\infty}\left(\tilde{\psi}_{T, i}^{*}(\omega)\left(K_{i} \tilde{y}_{T, i}(\omega)-\tilde{\psi}_{T, i}(\omega)+\eta_{i} K_{i}\left(j \omega \tilde{y}_{T, i}(\omega)+D_{2, i} \tilde{y}_{T, i}(\omega)+D_{3, i} \tilde{\psi}_{T, i}(\omega)\right)\right)\right. \\
\left.\left.+\left|\eta_{i} D_{1, i}\right|_{+} K_{i} \mid \tilde{y}_{T, i}(\omega)\right)\left.\right|^{2}\right) d \omega .
\end{array}
$$

Now, using Hypothesis (H3), there exist positive numbers $\varepsilon$ and $\rho_{y}$, both independent from $T$ and $\phi$, such that the open ball in $\mathbb{R}^{p}$ with centre 0 and radius $\rho_{y}$ is contained in $\mathcal{O}$ and such that, if

$$
\forall t \in[0, T], \quad\|y(t)\|<\rho_{y},
$$

then the following holds:

$$
\begin{aligned}
\left.\frac{1}{2 \pi} \int_{-\infty}^{+\infty}\left(\tilde{\psi}_{T}^{*}(\omega)\left(K \tilde{y}_{T}(\omega)-\tilde{\psi}_{T}(\omega)+\eta K\left(j \omega \tilde{y}_{T}(\omega)+D_{2} \tilde{y}_{T}(\omega)+D_{3} \tilde{\psi}_{T}(\omega)\right)\right)+\tilde{y}_{T}(\omega)\left|\eta D_{1}\right|_{+} K \tilde{y}_{T}(\omega)\right)\right) d \omega \\
=\frac{1}{2 \pi} \int_{-\infty}^{+\infty} \tilde{\psi}_{T}^{*}(\omega)\left(-I+\eta K D_{3}-\left(I+\eta\left(j \omega+D_{2}\right)\right) K H(j \omega)+H^{*}(j \omega)\left|\eta D_{1}\right|_{+} K H(j \omega)\right) \tilde{\psi}_{T}(\omega) d \omega \\
\left.\quad \text { using the identity } \tilde{y}_{T}(\omega)=-H(j \omega) \tilde{\psi}_{T}(\omega)\right) \\
\leq-\frac{\varepsilon}{2 \pi} \int_{-\infty}^{+\infty}\left\|\tilde{\psi}_{T}(\omega)\right\|^{2} d \omega=-\varepsilon \int_{0}^{+\infty}\left\|\psi_{T}(t)\right\|^{2} d t .
\end{aligned}
$$

It follows that

$$
\begin{aligned}
\frac{\varepsilon}{\|H\|_{\infty}^{2}} & \int_{0}^{+\infty}\left\|y_{T}(t)\right\|^{2} d t \leq \varepsilon \int_{0}^{+\infty}\left\|\psi_{T}(t)\right\|^{2} d t \\
\leq & \int_{0}^{T}\left(\psi_{T}^{*}(t) K\left(y(t)-y_{T}(t)\right)+\gamma(\|y(t)\|) y^{*}(t) K y(t)\right) d t+\sum_{i=0}^{p} \eta_{i} K_{i} \int_{0}^{y_{i}(0)} \psi_{i}(0, z) d z \\
& -\int_{0}^{T}\left(\psi_{T}^{*}(t) \eta K\left(\dot{y}_{T}(t)-\dot{y}(t)\right)+\left(y_{T}^{*}(t)-y^{*}(t)\right) \eta K D_{1}\left(y_{T}(t)-y(t)\right)+\psi_{T}^{*}(t) \eta K D_{2}\left(y_{T}(t)-y(t)\right)\right) d t .
\end{aligned}
$$


Due to (5) and (21)-(23), one may bound from above the previous expression by

$$
c_{3}\|\phi\|_{\mathcal{C}([-h, 0])}\left(\|\phi\|_{\mathcal{C}([-h, 0])}+\sup _{t \in[0, T]}\left\|y_{T}(t)\right\|\right)+c_{4} \sup _{t \in[0, T]} \gamma(\|y(t)\|) \int_{0}^{T}\left\|y_{T}(t)\right\|^{2} .
$$

To summarize, if (25) holds, then $y(t) \in \mathcal{O}$ for any $t \in[0, T]$, and

$\varepsilon \int_{0}^{+\infty}\left\|y_{T}(t)\right\|^{2} d t \leq c_{5}\left(\|\phi\|_{\mathcal{C}([-h, 0])}\left(\|\phi\|_{\mathcal{C}([-h, 0])}+\sup _{t \in[0, T]}\left\|y_{T}(t)\right\|\right)+\sup _{t \in[0, T]} \gamma(\|y(t)\|) \int_{0}^{T}\left\|y_{T}(t)\right\|^{2} d t\right)$.

Let us suppose that (25) holds, together with

$$
\forall t \in[0, T], c_{5} \gamma(\|y(t)\|) \leq \frac{\varepsilon}{2} .
$$

Then,

$$
\begin{aligned}
\int_{0}^{T}\left\|y_{T}(t)\right\|^{2} d t & \leq \frac{2 c_{5}}{\varepsilon}\|\phi\|_{\mathcal{C}([-h, 0])}\left(\|\phi\|_{\mathcal{C}([-h, 0])}+\sup _{t \in[0, T]}\left\|y_{T}(t)\right\|\right) \\
& =c_{6}\|\phi\|_{\mathcal{C}([-h, 0])}\left(\|\phi\|_{\mathcal{C}([-h, 0])}+\sup _{t \in[0, T]}\left\|y_{T}(t)\right\|\right) .
\end{aligned}
$$

We then deduces an estimate on $\dot{y}_{T}$ as follows:

$$
\begin{aligned}
\int_{0}^{T}\left\|\dot{y}_{T}(t)\right\|^{2} d t & \leq\left\|s\left(\sum_{l=0}^{L} C_{l} e^{-h_{l} s}\right)\left(s I-\sum_{l=0}^{L} A_{l} e^{-h_{l} s}\right)^{-1} B\right\|_{\infty}^{2} \int_{0}^{T}\left\|\psi_{T}(t)\right\|^{2} d t \\
& \leq\left\|s\left(\sum_{l=0}^{L} C_{l} e^{-h_{l} s}\right)\left(s I-\sum_{l=0}^{L} A_{l} e^{-h_{l} s}\right)^{-1} B\right\|_{\infty}^{2}\|K\|^{2} \int_{0}^{T}\|y(t)\|^{2} d t \\
& \leq c_{7}\|\phi\|_{\mathcal{C}([-h, 0])}\left(\|\phi\|_{\mathcal{C}([-h, 0])}+\sup _{t \in[0, T]}\left\|y_{T}(t)\right\|\right),
\end{aligned}
$$

using (23) and (27). One infers that (25), (26) imply, for any $t \in[0, T]$ :

$$
\frac{1}{2}\left\|y_{T}(t)\right\|^{2} \leq \int_{0}^{T} y_{T}^{T}(t) \dot{y}_{T}(t) d t+\frac{1}{2}\left\|y_{T}(0)\right\|^{2} \leq \sqrt{c_{6} c_{7}}\|\phi\|_{\mathcal{C}([-h, 0])}\left(\|\phi\|_{\mathcal{C}([-h, 0])}+\sup _{t \in[0, T]}\left\|y_{T}(t)\right\|\right),
$$

as $y_{T}(0)=0$ and by use of Cauchy-Schwarz inequality. The following inequality is hence true:

$$
\left(\sup _{t \in[0, T]}\left\|y_{T}(t)\right\|\right)^{2}-2 \sqrt{c_{6} c_{7}}\|\phi\|_{\mathcal{C}([-h, 0])}\left(\sup _{t \in[0, T]}\left\|y_{T}(t)\right\|\right)-2 \sqrt{c_{6} c_{7}}\|\phi\|_{\mathcal{C}([-h, 0])}^{2} \leq 0,
$$

from which one concludes that:

$T$ is such that $(25),(26)$ hold $\Rightarrow \sup _{t \in[0, T]}\left\|y_{T}(t)\right\|, \sup _{t \in[0, T]}\|y(t)\| \leq c_{8}\|\phi\|_{\mathcal{C}([-h, 0])}$.

Now, let $\rho_{x}>0$ be such that (recall that $\gamma(z) \rightarrow 0$ when $z \rightarrow 0$ )

$$
\rho_{x} \leq \frac{\rho_{y}}{2 c_{8}} \quad \text { and } \quad \forall z \in \mathbb{R}^{+}, z \leq c_{8} \rho_{x} \Rightarrow c_{5} \gamma(z) \leq \frac{\varepsilon}{4} .
$$


Choose $\phi \in \mathcal{C}\left([-h, 0] ; \mathbb{R}^{n}\right)$ with $\|\phi\|_{\mathcal{C}([-h, 0])}<\rho_{x}$. Performing the previous computations shows that the following inequalities are verified for $T=0$ and as long as (25), (26) hold:

$$
\begin{gathered}
\sup _{t \in[0, T]}\|y(t)\| \leq c_{8}\|\phi\|_{\mathcal{C}([-h, 0])} \leq c_{8} \rho_{x}, \quad \text { so } c_{5} \sup _{t \in[0, T]} \gamma(\|y(t)\|) \leq \frac{\varepsilon}{4}<\frac{\varepsilon}{2}, \\
\sup _{t \in[0, T]}\|y(t)\| \leq c_{8} \rho_{x} \leq \frac{\rho_{y}}{2}<\rho_{y} .
\end{gathered}
$$

From the continuity of $y$, one deduces that (25), (26) hold for any $T>0$, so

$$
\|\phi\|_{\mathcal{C}([-h, 0])}<\rho_{x} \Rightarrow \forall T \geq 0, \sup _{t \in[0, T]}\|y(t)\| \leq c_{8} \rho_{x} .
$$

This in turn implies, by (27), (28), that $y, \dot{y} \in L^{2}(0,+\infty)$. One concludes that $y(t) \rightarrow 0$ when $t \rightarrow+\infty$, which expresses the uniform local asymptotic stability of the origin.

When $\gamma \equiv 0$ and $\mathcal{O}=\mathbb{R}^{p}$, one may take $\rho_{y}=\rho_{x}=+\infty$.

- We now remove the assumption that $\eta \geq 0$. This part of the proof is similar to the analog enlargement of Popov criterion to nonpositive slopes $\eta$, see [2]. By hypothesis, one has:

$$
I-\eta K D_{3}+\left(I+\eta\left(s I+D_{2}\right)\right) K H(s)-H^{*}(s) \sup \left\{\eta D_{1} ; \frac{|\eta|-\eta}{2} K\left(D_{2}+K D_{3}\right)\right\} K H(s) \quad \text { is SPR . }
$$

Consider [31]

$$
\varphi(t, y) \stackrel{\text { def }}{=} K y-\psi(t, y)
$$

Let us choose the input $\varphi_{i}$ instead of $\psi_{i}$ when $\eta_{i} \leq 0$, and write that (1) is equivalent to

$$
\dot{x}=\sum_{l=0}^{L} \hat{A}_{l} x\left(t-h_{l}\right)+\hat{B} u, \quad u=-\hat{\psi}(t, y(t)) \stackrel{\text { def }}{=}-[\hat{J} \varphi(t, y(t))+(I-\hat{J}) \psi(t, y(t))], \quad y=\sum_{l=0}^{L} C_{l} x\left(t-h_{l}\right),
$$

where $\hat{I}, \hat{J}, \hat{A}_{l}, 0 \leq l \leq L, \hat{B}$ are defined by:

$$
\hat{I} \stackrel{\text { def }}{=} \operatorname{sgn} \eta, \hat{J} \stackrel{\text { def }}{=}(I-\operatorname{sgn} \eta) / 2, \hat{A}_{l}=A_{l}-B \hat{J} K C_{l}, \hat{B}=B \hat{I} .
$$

The following properties will be used repeatedly:

$$
\hat{J}^{2}=\hat{J}=-\hat{I} \hat{J}, 2 \hat{J}+\hat{I}=\hat{I}^{2}=I_{p} .
$$

If $\psi$ verifies Hypothesis (H1), then the same holds for $\hat{\psi}$, as

$$
\hat{\psi}(t, y)^{T}(\hat{\psi}(t, y)-K y)=\psi(t, y)^{T}(\psi(t, y)-K y) .
$$

As

$$
\begin{aligned}
& \eta_{i}\left(\int_{0}^{y_{i}} \frac{\partial \psi_{i}}{\partial t}(t, z) d z-D_{1, i} y_{i}^{2}-D_{2, i} y_{i} \psi_{i}\left(t, y_{i}\right)-D_{3, i} \psi_{i}\left(t, y_{i}\right)^{2}\right) \\
= & -\eta_{i}\left(\int_{0}^{y_{i}} \frac{\partial \varphi_{i}}{\partial t}(t, z) d z+\left(D_{1, i}+D_{2, i} K_{i}+D_{3, i} K_{i}^{2}\right) y_{i}^{2}-\left(D_{2, i}+2 D_{3, i} K_{i}\right) y_{i} \varphi_{i}\left(t, y_{i}\right)+D_{3, i} \varphi_{i}\left(t, y_{i}\right)^{2}\right)
\end{aligned}
$$

(H3) leads to a similar property on $\hat{\psi}$, with the values:

$$
\hat{\eta} \stackrel{\text { def }}{=} \hat{I} \eta=|\eta|, \hat{D}_{1} \stackrel{\text { def }}{=} \hat{I} D_{1}-\hat{J} K\left(D_{2}+K D_{3}\right), \hat{D}_{2} \stackrel{\text { def }}{=} D_{2}+2 \hat{J} K D_{3}, \hat{D}_{3} \stackrel{\text { def }}{=} \hat{I} D_{3} .
$$


Now, in order to achieve the proof, it is sufficient to show that if formula (29) holds, then the modified system verifies a condition analog to $(7)$ with the new value $\hat{\eta} \geq 0$, that is

$$
I-\hat{\eta} K \hat{D}_{3}+\left(I+\hat{\eta}\left(s I+\hat{D}_{2}\right)\right) K \hat{H}(s)-\hat{H}^{*}(s)\left|\hat{\eta} \hat{D}_{1}\right|_{+} K \hat{H}(s) \quad \text { is SPR , }
$$

where

$$
\hat{H}(s) \stackrel{\text { def }}{=}\left(\sum_{l=0}^{L} C_{l} e^{-h_{l} s}\right)\left(s I-\sum_{l=0}^{L} \hat{A}_{l} e^{-h_{l} s}\right)^{-1} \hat{B} .
$$

As a matter of fact, the first part of the proof may then be applied to the transformed system, and this concludes the proof of Theorem 1. It hence remains to prove (31). This is done with the help of the following Lemma.

Lemma 6. The following identity holds:

$$
\hat{H}(s)=(I+H(s) \hat{J} K)^{-1} H(s) \hat{I}=H(s)(I+\hat{J} K H(s))^{-1} \hat{I} .
$$

Proof. Lemma 6 is a consequence of the feedback structure involved when changing the input of the linear plant. Indeed, the new input $\hat{\psi}$ being defined as above, one has:

$$
\begin{aligned}
y & =-H \psi=-H(\hat{J} \psi+(I-\hat{J}) \psi)=-H(\hat{J}(K y-\varphi)+(I-\hat{J}) \psi) \\
& =-H(\hat{J} K y-\hat{J} \hat{\psi}+(I-\hat{J}) \hat{\psi})=-H(\hat{I} \hat{\psi}+\hat{J} K y),
\end{aligned}
$$

and finally:

$$
(I+H \hat{J} K) y=-H \hat{I} \hat{\psi},
$$

which gives the 1st equality. Deduction of the 2nd equality is straightforward.

Applying Lemma 6 one gets

$$
\begin{array}{r}
2\left(I-\hat{\eta} K \hat{D}_{3}\right)+\left(I+\hat{\eta}\left(s I+\hat{D}_{2}\right)\right) K \hat{H}(s)+\hat{H}^{*}(s) K\left(I+\hat{\eta}\left(s^{*} I+\hat{D}_{2}\right)\right)-2 \hat{H}^{*}(s)\left|\hat{\eta} \hat{D}_{1}\right|_{+} K \hat{H}(s) \\
=\hat{I}\left(I+H^{*}(s) K \hat{J}\right)^{-1} G(s)(I+\hat{J} K H(s))^{-1} \hat{I},
\end{array}
$$

where

$$
\begin{aligned}
G(s)= & 2\left(I+H^{*}(s) K \hat{J}\right)\left(I-\hat{\eta} K \hat{D}_{3}\right)(I+\hat{J} K H(s))+\left(I+H^{*}(s) K \hat{J}\right) \hat{I}\left(I+\hat{\eta}\left(s I+\hat{D}_{2}\right)\right) K H(s) \\
& +H^{*}(s) K\left(I+\hat{\eta}\left(s^{*} I+\hat{D}_{2}\right)\right) \hat{I}(I+\hat{J} K H(s))-2 H^{*}(s) K\left|\hat{\eta} \hat{D}_{1}\right|_{+} H(s) \\
= & 2\left(I-\hat{\eta} K \hat{D}_{3}\right)+\left(2 \hat{J}+\hat{I}+\hat{\eta}\left(-2 \hat{J} K \hat{D}_{3}+\hat{I}\left(s I+\hat{D}_{2}\right)\right)\right) K H(s) \\
& +H^{*}(s) K\left(I+\hat{\eta}\left(-2 \hat{J} K \hat{D}_{3}+\hat{I}\left(s^{*} I+\hat{D}_{2}\right)\right)\right) \\
& +H^{*}(s)\left(2 K^{2} \hat{J}(\hat{I}+\hat{J})+\hat{\eta} K^{2} \hat{J} \hat{I}\left(s+s^{*}\right)+2 K\left(-\left|\hat{\eta} \hat{D}_{1}\right|_{+}+\hat{\eta} K \hat{I} \hat{J} \hat{D}_{2}-\hat{\eta} \hat{J}^{2} K^{2} \hat{D}_{3}\right)\right) H(s),
\end{aligned}
$$

using the fact that the (diagonal) matrices $K, \hat{\eta}, \hat{I}, \hat{J}, \hat{D}_{j}$ commute. From (30), one gets

$$
\begin{aligned}
G(s)=2\left(I-\eta K D_{3}\right)+(I+\eta(s I & \left.\left.+D_{2}\right)\right) K H(s)+H^{*}(s) K\left(I+\eta\left(s^{*} I+D_{2}\right)\right) \\
& -2 H^{*}(s)\left(\left|\eta\left(D_{1}+\hat{J} K\left(D_{2}+K D_{3}\right)\right)\right|_{+}-\eta \hat{J} K\left(D_{2}+K D_{3}\right)\right) K H(s),
\end{aligned}
$$

so (29) and (31) are equivalent. This achieves the proof of Theorem 1. 


\section{References}

[1] M.A. Aizerman, On the effect of nonlinear functions of several variables on the stability of automatic control problems, Avtomat. i telemeh., 8, 1, 20-29, 1947 [Russian]

[2] M.A. Aizerman, F.R. Gantmacher, Absolute stability of regulator systems, Holden-Day Inc., 1964

[3] N.E. Barabanov, New frequency criteria for absolute stability and instability of automatic-control systems with nonstationary nonlinearities, Differential Equations 25, no 4, 367-373, 1989

[4] N.E. Barabanov, A.Kh. Gelig, G.A. Leonov, A.L. Likhtarnikov, A.S. Matveev, V.B. Smirnova, A.L. Fradkov, The frequency theorem (the Yakubovich-Kalman lemma) in control theory. Automat. Remote Control 57 (1996), no 10, part 1, 1377-1407, 1997

[5] G. Bertoni, C. Bonivento, E. Sarti, A graphical method for investigating the absolute stability of timevarying systems, Atti Accad. Sci. Ist. Bologna Cl. Sci. Fis. Rend. (12) 7, fasc. 1, 54-71, 1969/1970

[6] P.-A. Bliman, A.M. Krasnosel'skii, Popov absolute stability criterion for time-varying multivariable nonlinear systems, to appear in the Proc. of 5th Eur. Cont. Conf., Karlsruhe (Germany), 1999

[7] S. Boyd, L. El Ghaoui, E. Feron, V. Balakrishnan, Linear matrix inequalities in system and control theory, SIAM Studies in Applied Mathematics vol. 15, 1994

[8] C. Corduneanu, Absolute stability of some integro-differential systems. Ordinary differential equations (Proc. Conf., Math. Res. Center, Naval Res. Lab., Washington, D.C., 1971), Academic Press, New York, 55-70, 1972.

[9] C.A. Desoer, M. Vidyasagar, Feedback systems: input-output properties, Academic Press, 1975

[10] A.L. Fradkov, A.Yu. Pogromsky, Introduction to control of oscillation and chaos, World Scientific Publishers Co, 1998

[11] Lj.T. Grujić, P. Borne, J.C. Gentina, Matrix approaches to the absolute stability of time-varying LuriePostnikov systems, Internat. J. Control 30, no 6, 967-980, 1979

[12] A. Halanay, Differential equations: stability, oscillations, time lags, Academic Press, New York-London, 1966

[13] A. Halanay, Vl. Răsvan, Approximations of delays by ordinary differential equations, Recent advances in differential equations, R. Conti ed., Academic Press, New York-London, 155-197, 1981

[14] H.H. Hul'chuk, M.M. Lychak, Absolute stability of nonlinear control systems with nonstationary nonlinearities and tachometer feedback, Soviet Automat. Control 5, no 4, 6-9, 1972

[15] H.K. Khalil, Nonlinear systems, Macmillan Publishing Company, 1992

[16] G.A. Leonov, Extension of Popov's frequency criterion for nonstationary nonlinearities, Automat. Remote Control 41 (1980), no 11, part 1, 1494-1499, 1981

[17] G.A. Leonov, I.M. Burkin, A.I. Shepeljavyi, Frequency methods in oscillation theory, Kluwer, 1996

[18] X.-J. Li, On the absolute stability of systems with time lags, Chinese Math. 4, 609-626, 1963

[19] X.X. Liao, Absolute stability of nonlinear control systems, Mathematics and its Applications (Chinese Series) 5, Kluwer Academic Publishers Group, Dordrecht; Science Press, Beijing, 1993

[20] M.R. Liberzon, New results on absolute stability of nonstationary controlled systems (survey), Automat. Remote Control 40 (1979), no 8, part 1, 1124-1140, 1980

[21] M.R. Liberzon, The inner feature of absolute stability of nonstationary systems. Automat. Remote Control 50, no 5, part 1, 608-613, 1989 
[22] K.S. Narendra, J.H. Taylor, Frequency domain criteria for absolute stability, Academic Press, New-York London, 1973

[23] J.R. Partington, K. Glover, H.J. Zwart, R.F. Curtain, $L_{\infty}$ approximation and nuclearity of delay systems, Systems Control Lett. 10, no 1, 59-65, 1988

[24] V.A. Pliss, Certain problems in the theory of stability in the whole, Publisher: Leningrad State University (LUG), 1958 [Russian]

[25] V.M. Popov, Absolute stability of nonlinear systems of automatic control, Automat. Remote Control, 22, 857-875, 1961

[26] V.M. Popov, A. Halanay, On the stability of nonlinear automatic control systems with lagging argument, Automat. Remote Control 23, 783-786, 1962

[27] E.S. Pyatnitskii, Absolute stability of nonstationary nonlinear systems. Automat. Remote Control, no $1,1-9,1970$

[28] K. Pyragas, Continuous control of chaos by self-controlling feedback, Phys. Lett. A 170, 421-428, 1992

[29] K. Pyragas, Control of chaos via extended delay feedback, Phys. Lett. A 206, no 5-6, 323-330, 1995

[30] Vl. Răsvan, Frequency domain stability criteria for time lag control systems with several nonlinearities. III. Linear and nonlinear systems with monotonic time varying gain, Rev. Roumaine Sci. Tech. Sér. Électrotech. Énergét. 17, 507-521, 1972

[31] Z.V. Rekasius, J.E. Gibson, Stability analysis of nonlinear control systems by the second method of Lyapunov, IRE Trans. Automatic Control AC-7, no 1, 3-15, 1962

[32] Z.V. Rekasius, J.R. Rowland, A stability criterion for feedback systems containing a single time-varying nonlinear element, IEEE Trans. Automatic Control, 352-354, 1965

[33] R.T. Rockafellar, Convex analysis, Princeton Mathematical Series No. 28, Princeton University Press, 1970

[34] W. Rudin, Real and complex analysis, 3rd edition, McGraw-Hill, 1987

[35] J.A. Walker, Stability of feedback systems involving time delays and a time-varying nonlinearity, Int. $J$. of Control 6, no 4, 365-372, 1967

[36] J.A. Walker, Liapunov functions for feed-back systems containing a single time-varying non-linearity, Int. J. of Control 7, no 2, 171-174. 1968

[37] J.L. Willems, Stability Theory of Dynamical Systems, Thomas Nelson ans Sons Ltd, 1970

[38] V.A. Yakubovich, The method of matrix inequalities in the theory of stability of non-linear controlled systems. III. Absolute stability of systems with hysteresis non-linearities, Autom. Remote Control 26, $753-763,1965$ 


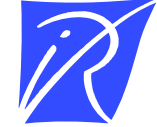

Unit'e de recherche INRIA Lorraine, Technopôle de Nancy-Brabois, Campus scientifique, 615 rue du Jardin Botanique, BP 101, 54600 VILLERS LÈS NANCY

Unit'e de recherche INRIA Rennes, Irisa, Campus universitaire de Beaulieu, 35042 RENNES Cedex

Unit'e de recherche INRIA Rhône-Alpes, 655, avenue de l'Europe, 38330 MONTBONNOT ST MARTIN

Unit'e de recherche INRIA Rocquencourt, Domaine de Voluceau, Rocquencourt, BP 105, 78153 LE CHESNAY Cedex

Unit'e de recherche INRIA Sophia-Antipolis, 2004 route des Lucioles, BP 93, 06902 SOPHIA-ANTIPOLIS Cedex

\section{Éditeur}

INRIA, Domaine de Voluceau, Rocquencourt, BP 105, 78153 LE CHESNAY Cedex (France)

http://www.inria.fr

ISSN 0249-6399 